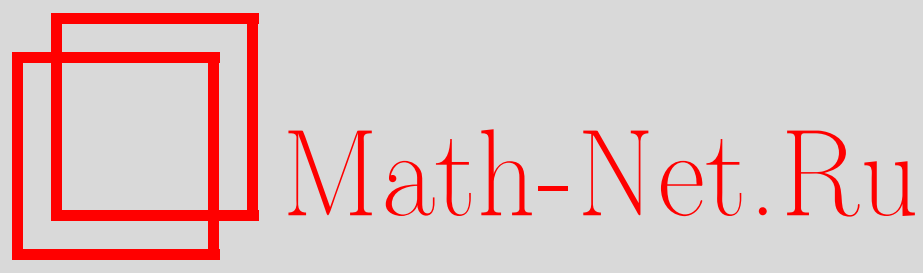

Н. Р. Садыков, Волновые функции и собственные значения носителей заряда в нанотрубке в окрестности точки Дирака при наличии продольного электрического поля, ТМФ, 2014, том 180, номер 3, 368-381

DOI: https://doi.org/10.4213/tmf8642

Использование Общероссийского математического портала Math-Net.Ru подразумевает, что вы прочитали и согласны с пользовательским соглашением http://www.mathnet.ru/rus/agreement

Параметры загрузки:

IP : 34.227 .88 .159

26 апреля 2023 г., 10:33:53

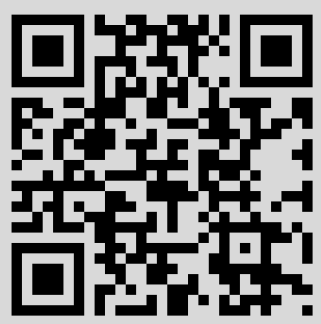


Том 180, № 3

сентябрь, 2014

\title{
ВОЛНОВЫЕ ФУНКЦИИ И СОБСТВЕННЫЕ ЗНАЧЕНИЯ НОСИТЕЛЕЙ ЗАРЯДА В НАНОТРУБКЕ В ОКРЕСТНОСТИ ТОЧКИ ДИРАКА ПРИ НАЛИЧИИ ПРОДОЛЬНОГО ЭЛЕКТРИЧЕСКОГО ПОЛЯ
}

\begin{abstract}
На основе гамильтониана для носителей тока в углеродных нанотрубках конечной длины при наличии продольного электрического поля получены собственные значения и собственные функции в окрестности точек Дирака (волновые функции, записанные по аналогии с двухкомпонентной волновой функцией Дирака, выражаются через полиномы Эрмита, а спектр является эквидистантным). В случае углеродных нанотрубок неограниченной длины решение выражается через функции Эрмита. На основе полученной волновой функции для удлиненной нанотрубки рассмотрена задача определения коэффициента прохождения носителей заряда через нанотрубку. Результаты по определению коэффициента прохождения также применимы к другим наночастицам, в частности к углеродным цепочкам и к нанолентам. Собственные значения и собственные функции нанотрубок конечной длины предлагается использовать при рассмотрении задачи генерации излучения в нелинейной среде на основе массива таких невзаимодействующих нанотрубок.
\end{abstract}

Ключевые слова: двухволновая волновая функция Дирака, массив нанотрубок, наноленты, коэффициент прохождения.

DOI: $10.4213 / \operatorname{tmf} 8642$

\section{1. ВВЕДЕНИЕ}

Квазичастицы в графене обладают линейным законом дисперсии вблизи точек Дирака [1], [2]. Это приводит к тому, что носители заряда формально описываются гамильтонианом дираковского типа, их волновая функция аналогична двухкомпонентной волновой функции Дирака, а свойства носителей заряда можно описать с помощью уравнения Дирака [2]-[4]. Сами точки Дирака находятся в вершинах шестиугольников на фазовой плоскости $\left(p_{x}, p_{z}\right)$ (границы шестиугольников задают область определения квазиимпульса - первую зону Бриллюэна), где электроны

*Национальный исследовательский ядерный институт "МИФИ" Снежинский физикотехнический институт, Снежинск, Челябинская обл., Россия.

E-mail: n.r.sadykov@rambler.ru. 
обладают большим волновым вектором. Если пренебречь процессами переброса между долинами, то в низкоэнергетическом приближении такое большое значение волнового вектора никак не влияет на транспорт, поэтому волновой вектор, фигурирующий в уравнении Дирака, отсчитывают от точек Дирака, а уравнение Дирака записывают для разных долин отдельно.

Данный подход широко используется при исследовании различных процессов (см., например, работы [5]-[11]). В работе [5] реализован однослойный графеновый $p-n$ переход. При измерении транспортных свойств в квантовом эффекте Холла выявлено новое плато проводимости, в полтора раза превышающее квант проводимости $2 e^{2} / h$ (как обычно, $h=2 \pi \hbar$ - постоянная Планка). В работе [6] изучается фокусировка электронного потока в графене в результате $p-n$ перехода. В работе [7] рассматривается задача распространения носителя заряда в графене в одномерном волноводе с потенциалом в виде ступенчатой функции. Отмечено, что в связи с бесщелевым характером спектра квазичастиц в графене электростатическое удержание частицы не является возможным. В работе [8] процесс распространения носителей заряда в гладком электростатическом потенциальном поле изучается по аналогии с одномодовой световедущей сердцевиной. В этом случае система уравнений Дирака может быть сведена к обобщенному уравнению гипергеометрического типа. В работе [9] по аналогии со статьей [8] рассмотрен случай осесимметричного потенциала. В работе [10] вычислена динамическая проводимость АА-упаковки биграфена в зависимости от частоты и с учетом химического потенциала, создаваемого электрическим зарядом. В работе [11] для двуслойной АА-упаковки биграфена предсказано наличие основных состояний, аналогичных случаю антиферромагнетизма. Подход, используемый в статьях [8], [9], является нетривиальным, если учесть распространенное мнение, что электростатическое удержание носителей заряда в графене невозможно из-за эффекта клейновского туннелирования [12]. Само клейновское туннелирование нашло интересное применение, например, к двуслойным углеродным нанотрубкам (УНТ) [13]. В работе [13] показано, что в двуслойном биграфене при $n-p-n$ переходе может происходить клейновское туннелирование электрона.

В настоящей работе по аналогии с работами [8], [9] исследуется процесс распространения носителей заряда в электростатическом потенциальном поле, создаваемом продольным электрическим полем в УНТ или в графеновой наноленте (ГНЛ). В основе подхода лежит тот факт, что волновая функция заряженной частицы представляется в виде произведения быстроосциллирующего экспоненциального множителя и медленно меняющейся функции от продольной координаты. Экспоненциальная часть волновой функции определяется значением волнового числа (импульса) частицы в точке Дирака. Медленно меняющаяся часть волновой функции удовлетворяет системе дифференциальных уравнений, которые получаются из разностных по аналогии с работой [14]. Полученные результаты можно использовать для определения коэффициента прохождения в удлиненных наночастицах [14] и для описания квантовых состояний в каскадных лазерах [15]-[17] на основе массива наночастиц. Результаты настоящей работы частично обобщают результаты статьи [18].

В разделе 2 воспроизведен вывод эффективного гамильтониана носителя заряда вблизи точки Дирака при наличии продольного электрического поля в удлиненных наночастицах (см., например, главы 2-4 в монографии [19]). В разделе 3 решено полученное безмассовое уравнение Дирака в продольном электрическом поле с усло- 
вием периодичности, соответствующим определенной хиральности. Качественно такая ситуация аналогична рассеянию квазичастицы в графене на потенциале, зависящем от одной координаты [20]. В частности, в случае потенциального барьера для определенных углов падения наблюдается эффект клейновского туннелирования. В разделе 4 полученное решение используется для вычисления коэффициента прохождения носителей заряда через массив невзаимодействующих УНТ, который моделируется электростатическим потенциалом. Отмечается, что массив УНТ можно использовать для генерации квазимонохроматического терагерцового излучения.

\section{2. ВЫВОД ВОЛНОВОГО УРАВНЕНИЯ В УДЛИНЕННЫХ ГРАФЕНОВЫХ НАНОЧАСТИЦАХ НА ОСНОВЕ МОДЕЛИ ДВУХТОЧЕЧНОЙ ФАЗОВОЙ ЯЧЕЙКИ}

Рассмотрим удлиненные графеновые наночастицы типа УНТ или ГНЛ. Воспользуемся обозначениями работ [21], [22], в которых для нанотрубок типа $(n, m)$ приведен полученный на основе двухточечной фазовой ячейки закон дисперсии, определяющий зонную структуру графена. Этот закон дисперсии имеет вид (см. рис. 1)

$$
\varepsilon\left(k_{\|}, k_{\perp}\right)= \pm \gamma_{0}\left|e^{i \sqrt{3} k_{z} a / 2}+2 \cos \frac{k_{\mathrm{s}} a}{2}\right| .
$$

Здесь $a=\sqrt{3} b, b=0.142$ нм - расстояние между центрами атомов углерода, величина $\gamma_{0} \approx 2.7$ э - интеграл перескока,

$$
k_{z}=k_{\|} \cos \theta-k_{\perp} \sin \theta, \quad k_{\mathrm{s}}=k_{x}=k_{\perp} \cos \theta+k_{\|} \sin \theta
$$

(вектор $\mathbf{k}_{\mathrm{s}}$ на рис. 1 направлен вдоль штриховой линии, т. е. вдоль оси $O X$ ), где $\theta-$ угол хиральности углеродной нанотрубки, $|\theta| \leqslant \pi / 6$, и

$$
\mathbf{k}=k_{\|} \frac{\mathbf{T}}{T}+k_{\perp} \frac{\mathbf{C}_{\mathrm{h}}}{C_{\mathrm{h}}}, \quad C_{\mathrm{h}}=a \sqrt{n^{2}+m^{2}+n m}, \quad T=\frac{\sqrt{3} C_{\mathrm{h}}}{d_{R}},
$$

$d_{R}-$ наибольший общий делитель чисел $2 n+m$ и $2 m+n$, при этом

$$
-\frac{\pi}{T} \leqslant k_{\|} \leqslant \frac{\pi}{T}, \quad k_{\perp}=\frac{2 \pi l}{C_{\mathrm{h}}}, \quad l=0,1, \ldots, N-1 .
$$

Целое число $l$ задает угловой момент электрона вдоль оси нанотрубки, а число $N=2\left(n^{2}+m^{2}+n m\right) / d_{R}$ есть число элементарных атомных ячеек, состоящих из двух атомов углерода, в области $\left|\mathbf{C}_{\mathrm{h}} \times \mathbf{T}\right|$.

При $\theta=0$ мы имеем случай нанотрубки $(n, 0)$ типа "зигзаг". В этом случае величина $k_{\mathrm{s}}=k_{\perp}$ характеризует азимутальную компоненту волнового вектора, а величина $k_{\|}$- продольную (параллельную оси нанотрубки). При $\theta=\pi / 2$ реализуется случай нанотрубки $(n, n)$ типа "кресло". В этом случае $k_{\mathrm{s}}=k_{\|}$характеризует продольную компоненту, а $k_{\perp}$ - азимутальную компоненту волнового вектора. 


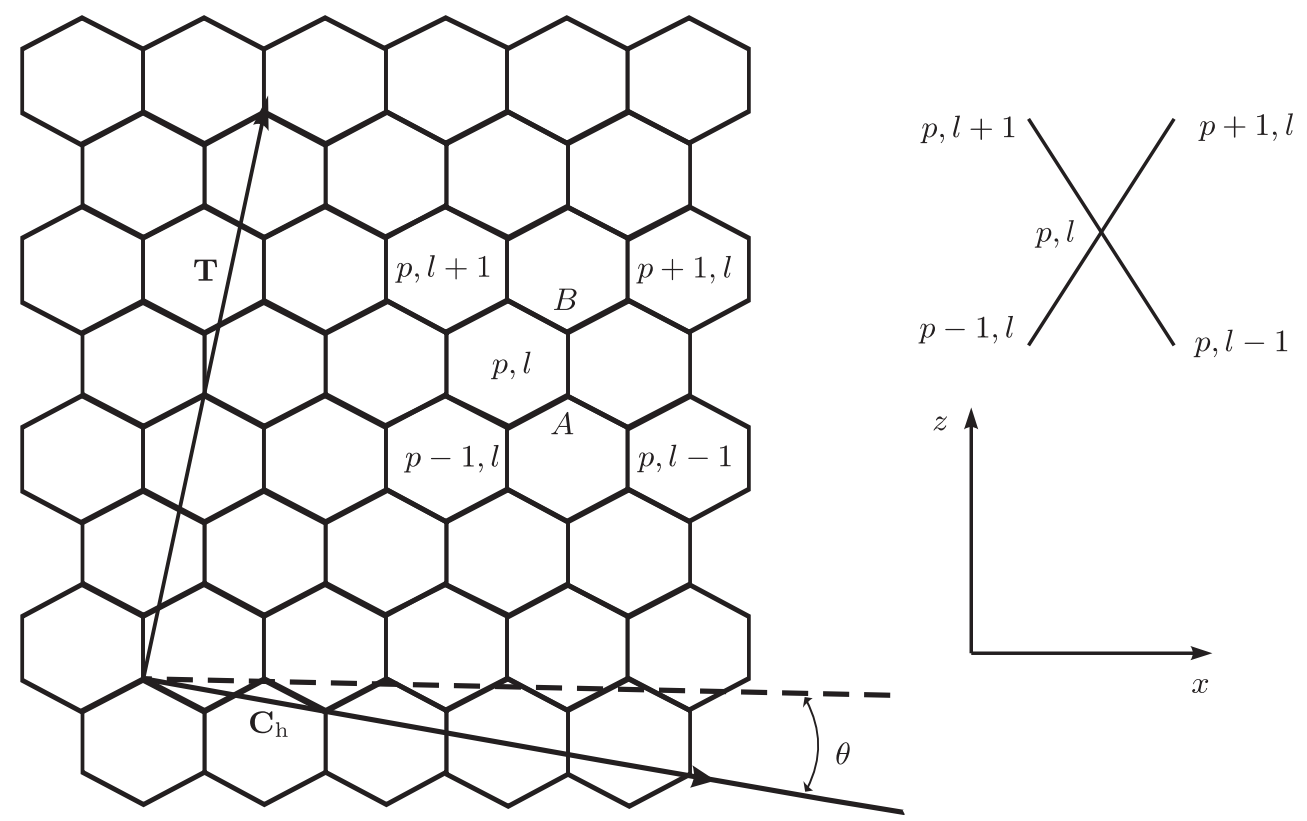

Рис. 1. Развернутый лист графита. Индексы $(p, l)$ определяют координаты центра двухточечной фазовой ячейки.
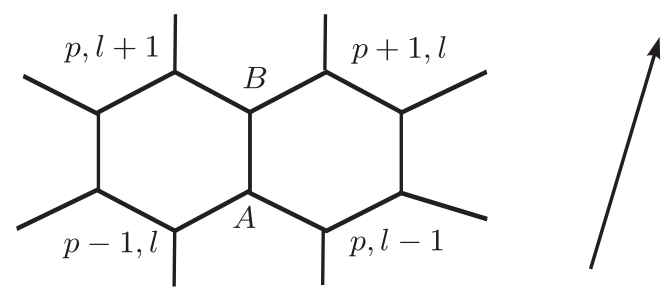

Рис. 2. Ориентация нанотрубки типа "зигзаг" в электрическом поле.

С учетом соотношений (см. рис. 1)

$$
\begin{gathered}
\cos \theta=\frac{2 n+m}{2 \sqrt{n^{2}+m^{2}+n m}}, \quad \sin \theta=\frac{\sqrt{3} m}{2 \sqrt{n^{2}+m^{2}+n m}}, \\
k_{z}=k_{\|} \cos \theta-k_{\perp} \sin \theta=\frac{2 n+m}{\sqrt{3} m} k_{\mathrm{s}} a-\frac{4 \pi l}{\sqrt{3} m a}
\end{gathered}
$$

закон дисперсии (1) можно переписать как

$$
\varepsilon= \pm \gamma_{0} \sqrt{1+4 \cos \frac{k_{\mathrm{s}} a}{2} \cos \frac{\sqrt{3} k_{z} a}{2}+4 \cos ^{2} \frac{k_{\mathrm{s}} a}{2}} .
$$

Рассмотрим двухточечную элементарную фазовую ячейку [19], [21] (см. рис. 2), в которой точки $A$ и $B$ принадлежат разным подрешеткам. В случае бесконечной 
графеновой плоскости для такой ячейки вводят две базисные функции Блоха

$$
\Psi_{A}=\frac{1}{\sqrt{Z}} \sum_{p, l} \chi_{A}\left(\mathbf{r}-\mathbf{r}_{p, l}^{A}\right), \quad \Psi_{B}=\frac{1}{\sqrt{Z}} \sum_{p, l} \chi_{B}\left(\mathbf{r}-\mathbf{r}_{p, l}^{B}\right),
$$

где индексы $p, l$ - целые числа, определяющие положение фазовой ячейки, $\mathbf{r}_{p, l}^{A}$ и $\mathbf{r}_{p, l}^{B}-$ радиус-векторы, характеризующие положение в ячейках точек $A$ и $B$, а $Z$ - полное число ячеек. Полная волновая функция для такого случая записывается как

$$
\Psi=\alpha \Psi_{A}+\beta \Psi_{B},
$$

где $\alpha$ и $\beta$ - постоянные величины. Для коэффициентов при функциях Блоха выполнено условие нормировки $|\alpha|^{2}+|\beta|^{2}=1$.

В случае, когда графеновой лист имеет конечные размеры (например, для УНТ или ГНЛ [23]), волновую функцию нельзя выразить через функции Блоха. Представим волновую функцию в виде линейной комбинации атомных орбиталей:

$$
\Psi=\sum_{p, l}\left(\alpha_{p, l} \chi_{A}\left(\mathbf{r}-\mathbf{r}_{p, l}^{A}\right)+\beta_{p, l} \chi_{B}\left(\mathbf{r}-\mathbf{r}_{p, l}^{B}\right)\right),
$$

где $\alpha_{p, l}$ и $\beta_{p, l}$ - величины, зависящие от расположения ячейки (от индексов $p, l$ ). Также справедливо условие нормировки

$$
\sum_{p, l}\left(\left|\alpha_{p, l}\right|^{2}+\left|\beta_{p, l}\right|^{2}\right)=1
$$

Величины $\alpha_{p, l}$ и $\beta_{p, l}$ суть функции, заданные на сетке, и в случае графенового листа больших размеров можно перейти от функций, заданных на сетке, к непрерывным функциям, а разностным операторам поставить в соответствие дифференциальные операторы [24] по аналогии с работами [14], [25]-[27].

Теперь для нанотрубок рассмотрим случай, когда присутствует постоянное электрическое поле $\mathbf{W}$, параллельное вектору $\mathbf{T}$ (см. рис. 2). Тогда получаем секулярное уравнение второго порядка для законов дисперсии $\pi$-электронов: мы имеем

$$
\begin{gathered}
\widehat{H} \Psi=E \Psi, \quad \widehat{H}=\widehat{H}_{0}+\widehat{V} \\
\widehat{H}_{0} \chi_{A}=E_{0} \chi_{A}, \quad \widehat{H}_{0} \chi_{B}=E_{0} \chi_{B}, \quad \widehat{V}=-\mathrm{eWr}=-\mathrm{e} W_{0} \xi,
\end{gathered}
$$

где $\xi=x \sin \theta+z \cos \theta$ - координата, которая отсчитывается вдоль оси нанотрубки. При этом

$$
\begin{gathered}
-\left(\varepsilon+\mathrm{e} W_{0} \xi_{p, l}^{A}\right) \alpha_{p, l}+\gamma_{0}\left(\beta_{p, l}+\beta_{p-1, l}+\beta_{p, l-1}\right)=0 \\
\gamma_{0}\left(\alpha_{p, l}+\alpha_{p+1, l}+\alpha_{p, l+1}\right)-\left(\varepsilon+\mathrm{e} W_{0} \xi_{p, l}^{B}\right) \beta_{p, l}=0
\end{gathered}
$$

где $\varepsilon=E-E_{0}$. При выводе системы (3) предполагается, что на величину интеграла перескока не влияет оператор возмущения $\widehat{V}$. В приведенных выше уравнениях через е $>0$ обозначена абсолютная величина заряда электрона, $\xi_{p, l}^{A}$ и $\xi_{p, l}^{B}-$ продольные (отсчитываемые вдоль вектора $\mathbf{T}$ на рис. 1) координаты узловых точек $A$ и $B$ ячейки $(p, l)$. 
Из уравнений (3) следует система разностных уравнений для коэффициентов при функциях Блоха:

$$
\begin{aligned}
& \left(\varepsilon+\mathrm{e} W_{0} \xi_{p, l}^{A}\right)^{2} \alpha_{p, l}=\gamma_{0}^{2}\left(3 \alpha_{p, l}+\sum_{\tilde{p}, \tilde{l}} \alpha_{\tilde{p}, \tilde{l}}\right) \\
& \left(\varepsilon+\mathrm{e} W_{0} \xi_{p, l}^{B}\right)^{2} \beta_{p, l}=\gamma_{0}^{2}\left(3 \beta_{p, l}+\sum_{\tilde{p}, \tilde{l}} \beta_{\tilde{p}, \tilde{l}}\right)
\end{aligned}
$$

где индексы суммирования $(\tilde{p}, \tilde{l})$ пробегают значения (см. рис. 1$)$

$$
(p, l+1), \quad(p, l-1), \quad(p+1, l), \quad(p-1, l), \quad(p-1, l+1), \quad(p+1, l-1) .
$$

В случае бесконечно большой графеновой плоскости при отсутствии постоянного поля решения уравнений (4) представим в виде

$$
\alpha_{p, l} \sim e^{i \sqrt{3} b k_{x} n_{x}+i 3 b k_{z} n_{z}}, \quad \beta_{p, l} \sim e^{i \sqrt{3} b k_{x} n_{x}+i 3 b k_{z} n_{z}},
$$

где $n_{x}, n_{z}=0, \pm 1, \pm 2, \ldots$ и $\mathbf{k}=\left(k_{x}, k_{z}\right)=\mathbf{p} / \hbar, \mathbf{p}-$ импульс электрона. Тогда получаем закон дисперсии для УНТ типа "зигзаг"

$$
\varepsilon^{2}=\gamma_{0}^{2}\left(1+4 \cos \frac{k_{x} a}{2} \cos \frac{\sqrt{3} k_{z} a}{2}+4 \cos ^{2} \frac{k_{x} a}{2}\right) .
$$

Этот закон дисперсии с учетом уравнений (4) при $W_{0}=0$ может быть записан в альтернативном виде

$$
\varepsilon^{2}=\gamma_{0}^{2}\left(3+\sum_{\tilde{p}, \tilde{l}} e^{i \mathbf{k}\left(\mathbf{r}_{\tilde{p}, \tilde{l}}-\mathbf{r}_{p, l}\right)}\right)
$$

где $\varepsilon=0$ на поверхности Ферми (при $\mathbf{k}=\mathbf{k}_{\mathrm{F}}$ ).

По аналогии с работами [25], [28]-[30], в которых использован метод временно́й дисперсии, будем искать решение уравнений (4) в виде интеграла Фурье:

$$
\begin{aligned}
& \alpha_{p l}=\alpha\left(\mathbf{r}_{p, l}\right)=\iiint \tilde{\alpha}(\mathbf{k}) e^{i \mathbf{k} \mathbf{r}_{p, l}} d^{3} \mathbf{k}, \\
& \beta_{p l}=\beta\left(\mathbf{r}_{p, l}\right)=\iiint \tilde{\beta}(\mathbf{k}) e^{i \mathbf{k r}_{p, l}} d^{3} \mathbf{k} .
\end{aligned}
$$

Рассмотрим первое из этих уравнений. В окрестности точки Дирака $\mathbf{k}_{\mathrm{F}}$ [1] выделим быстроосциллирующую часть функции $\alpha_{p l}$ :

$$
\alpha_{p, l}=\tilde{\alpha}\left(\mathbf{r}_{p, l}\right) e^{i \mathbf{k}_{\mathrm{F}} \mathbf{r}_{p, l}}, \quad \tilde{\alpha}\left(\mathbf{r}_{p, l}\right)=\iiint \tilde{\alpha}(\mathbf{k}) e^{i\left(\mathbf{k}-\mathbf{k}_{\mathrm{F}}\right) \mathbf{r}_{p, l}} d^{3} \mathbf{k} .
$$

Следует отметить, что вблизи точки Дирака $\mathbf{k}_{\mathrm{F}}$ закон дисперсии квазичастиц (5) можно преобразовать к линейному виду [1]

$$
\varepsilon^{2}=v_{\mathrm{F}}^{2}\left|\mathbf{p}-\mathbf{p}_{\mathrm{F}}\right|^{2}, \quad v_{\mathrm{F}}=\frac{\sqrt{3} \gamma_{0} a}{2 \hbar} ;
$$

здесь $v_{\mathrm{F}}-$ значение скорости электрона на поверхности Ферми. 
Аналогично получаем для второго уравнения в (7)

$$
\beta_{p, l}=\beta\left(\mathbf{r}_{p, l}\right)=\left\{\iiint \tilde{\beta}(\mathbf{k}) e^{i\left(\mathbf{k}-\mathbf{k}_{\mathrm{F}}\right) \mathbf{r}_{p, l}} d^{3} \mathbf{k}\right\} e^{i \mathbf{k}_{\mathrm{F}} \mathbf{r}_{p, l}} .
$$

В окрестности точки $\mathbf{k}_{\mathrm{F}}$ выражение в скобках в правой части первого из уравнений (4) с учетом формул (8) преобразуем к виду

$$
3 \alpha_{p, l}+\sum_{\tilde{p}, \tilde{l}} \alpha_{\tilde{p}, \tilde{l}}=\left\{\iiint \tilde{\alpha}(\mathbf{k})\left(3 e^{i\left(\mathbf{k}-\mathbf{k}_{\mathrm{F}}\right) \mathbf{r}_{p, l}}+\sum_{\tilde{p}, \tilde{l}} e^{i\left(\mathbf{k r}_{\tilde{p}, \tilde{l}}-\mathbf{k}_{\mathrm{F}} \mathbf{r}_{p, l}\right)}\right) d^{3} \mathbf{k}\right\} e^{i \mathbf{k}_{\mathrm{F}} \mathbf{r}_{p, l}} .
$$

Из этого уравнения после несложных, но относительно громоздких выкладок получаем формулу (П.4) приложения, из которой по аналогии с рассуждениями из работ [25]-[27] выводим соотношение

$$
3 \alpha_{p, l}+\sum_{\tilde{p}, \tilde{l}} \alpha_{\tilde{p}, \tilde{l}}=-\left.\frac{3 a^{2}}{4} \Delta_{\perp} \tilde{\alpha}(\mathbf{r})\right|_{\mathbf{r}=\mathbf{r}_{p, l}} e^{i \mathbf{k}_{\mathrm{F}} \mathbf{r}_{p, l}},
$$

где $\Delta_{\perp}=\partial^{2} / \partial x^{2}+\partial^{2} / \partial z^{2}$ - двумерный лапласиан на поверхности графена, а величина $v_{\mathrm{F}}$ определена в $(9)$.

Аналогично получаем для $\beta_{p, l}$ уравнение

$$
3 \beta_{p, l}+\sum_{\tilde{p}, \tilde{l}} \beta_{\tilde{p}, \tilde{l}}=-\left.\frac{3 a^{2}}{4} \Delta_{\perp} \tilde{\beta}(\mathbf{r})\right|_{\mathbf{r}=\mathbf{r}_{p, l}} e^{i \mathbf{k}_{\mathrm{F}} \mathbf{r}_{p, l}},
$$

Из уравнений (4) с учетом формул (11) и (12) вытекают уравнения

$$
\begin{aligned}
& \left(\varepsilon+\mathrm{e} W_{0} \xi\right)^{2} \tilde{\alpha}=-v_{\mathrm{F}}^{2} \hbar^{2} \Delta_{\perp} \tilde{\alpha} \\
& \left(\varepsilon+\mathrm{e} W_{0} \xi\right)^{2} \tilde{\beta}=-v_{\mathrm{F}}^{2} \hbar^{2} \Delta_{\perp} \tilde{\beta}
\end{aligned}
$$

где $\tilde{\alpha}=\tilde{\alpha}(\mathbf{r}), \tilde{\beta}=\tilde{\beta}(\mathbf{r})$ и $\xi=\left(\xi_{p, l}^{A}+\xi_{p, l}^{B}\right) / 2$. Таким образом, если рассматривать коэффициенты $\alpha_{p, l}$ и $\beta_{p, l}$ в (2) как непрерывные функции от $\mathbf{r}$, то с учетом соотношений (8) их можно представить в виде

$$
\alpha(\mathbf{r})=\tilde{\alpha}(\mathbf{r}) e^{i \mathbf{k}_{\mathrm{F}} \mathbf{r}}, \quad \beta(\mathbf{r})=\tilde{\beta}(\mathbf{r}) e^{i \mathbf{k}_{\mathrm{F}} \mathbf{r}},
$$

где $\tilde{\alpha}(\mathbf{r})$ и $\tilde{\beta}(\mathbf{r})$ удовлетворяют уравнениям (13). Решения (14) уравнений (2) получены для неограниченного графенового листа из предположения, что коэффициенты разложения в (2) описывают состояния вблизи поверхности Ферми (точки Дирака).

Решения уравнений (13) в одномерном случае (когда лапласиан зависит только от одной координаты) для УНТ или ГНЛ конечной длины можно выразить через полиномы Эрмита, а в случае "бесконечной длины" - через функции Эрмита. Системе уравнений (13) можно придать вид уравнений Дирака для безмассовой частицы, где величины $\tilde{\alpha}$ и $\tilde{\beta}$ выполняют роль двух компонент спиновой функции [2]-[4]. В этом случае из (13) следует, что

$$
\widehat{H} \Phi=\varepsilon \Phi, \quad \Phi=\left(\begin{array}{c}
\tilde{\alpha} \\
\tilde{\beta}
\end{array}\right), \quad \widehat{H}=v_{\mathrm{F}} \hat{\boldsymbol{\sigma}} \hat{\mathbf{p}}_{\perp}-\mathrm{e} W_{0} \xi
$$


где

$$
\hat{\boldsymbol{\sigma}}=\left(\hat{\sigma}_{x}, \hat{\sigma}_{y}\right), \quad \hat{\sigma}_{x}=\left(\begin{array}{cc}
0 & 1 \\
1 & 0
\end{array}\right), \quad \hat{\sigma}_{y}=\left(\begin{array}{cc}
0 & -i \\
i & 0
\end{array}\right)
$$

при этом

$$
\begin{aligned}
& \hat{\boldsymbol{\sigma}}=\left(\hat{\sigma}_{\xi}, \hat{\sigma}_{\eta}\right), \quad \hat{\sigma}_{\xi}=\mathbf{e}_{\xi} \boldsymbol{\sigma}, \quad \hat{\sigma}_{\eta}=\mathbf{e}_{\eta} \boldsymbol{\sigma}, \\
& \hat{\mathbf{p}}_{\perp}=\left(\hat{p}_{\xi}, \hat{p}_{\eta}\right), \quad \hat{p}_{\xi}=i \hbar \frac{\partial}{\partial \xi}, \quad \hat{p}_{\eta}=-i \hbar \frac{\partial}{\partial \eta},
\end{aligned}
$$

где $\xi=x \sin \theta+z \cos \theta-$ координата, которая отсчитывается вдоль оси нанотрубки (вдоль вектора $\mathbf{T}$ на рис. 1), и $\eta=x \cos \theta-z \sin \theta$ - перпендикулярная оси нанотрубки координата (вдоль вектора $\mathbf{C}_{\mathrm{h}}$ на рис. 1), $\mathbf{e}_{\xi}$ и $\mathbf{e}_{\eta}$ - базисные векторы, направленные вдоль координатных осей $O \xi$ и $O \eta$. При выводе уравнения (15) учтено, что в окрестности поверхности Ферми имеет место разложение (9) для закона дисперсии носителей тока в зоне проводимости (см. работу [1]).

\section{3. КВАНТОВЫЕ СОСТОЯНИЯ ВБЛИЗИ ПОВЕРХНОСТИ ФЕРМИ В ГРАФЕНОВЫХ СТРУКТУРАХ}

Полученные в предыдущем разделе уравнения позволяют найти коэффициент прохождения в УНТ или ГНЛ с металлическим типом зонной структуры. Данная задача является актуальной, поскольку основной вклад в проводимость вносят именно электроны с энергией в окрестности точки Дирака. Волновое число $\mathbf{k}_{\mathrm{F}}$ в этой точке определяет быстроосциллирующую часть полной волновой функции, а решение уравнений (13) - амплитуду волны, слабо зависящей от продольной координаты. Эти величины позволяют вычислить коэффициент прохождения [14]. Рассмотрим задачу более подробно.

Применим уравнения (13) к нанотрубке типа "зигзаг” (в случае $\theta=0$ на рис. 1). Пусть $R$ - радиус УНТ. Тогда в (13) поперечная составляющая оператора Лапласа дает решение $\tilde{\alpha} \sim e^{i \nu \varphi}, \nu=0, \pm 1, \pm 2, \ldots$. Соответственно поправка к энергии имеет вид $|\Delta E| \sim v_{\mathrm{F}} \hbar / R \sim 1$ эВ. Поскольку в дальнейшем нас будет интересовать приближение $|\Delta E| \leqslant 1$ эВ при $z=0$, на величину коэффициента прохождения будут оказывать влияние поперечные моды с $\nu \sim 1$. Поэтому систему уравнений (13) преобразуем к виду

$$
\begin{aligned}
& \frac{\partial^{2} \tilde{\alpha}}{\partial \xi^{2}}+\left(\left(\frac{\varepsilon+\mathrm{e} W_{0} \xi}{v_{\mathrm{F}} \hbar}\right)^{2}-\frac{\nu^{2}}{R^{2}}\right) \tilde{\alpha}=0 \\
& \frac{\partial^{2} \tilde{\beta}}{\partial \xi^{2}}+\left(\left(\frac{\varepsilon+\mathrm{e} W_{0} \xi}{v_{\mathrm{F}} \hbar}\right)^{2}-\frac{\nu^{2}}{R^{2}}\right) \tilde{\beta}=0 .
\end{aligned}
$$

Приведем эту систему обобщенных уравнений гипергеометрического типа к уравнениям гипергеометрического типа [31]: пусть

$$
\begin{gathered}
\tilde{\alpha}=Y_{\alpha} \exp \left( \pm i \frac{\left(\varepsilon+\mathrm{e} W_{0} \xi\right)^{2}}{2 \mathrm{e} W_{0} v_{\mathrm{F}} \hbar}\right) \\
\frac{\partial^{2} Y_{\alpha}}{\partial \xi^{2}} \pm 2 i \frac{\varepsilon+\mathrm{e} W_{0} \xi}{v_{\mathrm{F}} \hbar} \frac{\partial Y_{\alpha}}{\partial \xi}+\left( \pm i \frac{\mathrm{e} W_{0}}{v_{\mathrm{F}} \hbar}-\frac{\nu^{2}}{R^{2}}\right) Y_{\alpha}=0
\end{gathered}
$$


Решения уравнений (18) выражаются через функцию Эрмита:

$$
Y_{\alpha}(\xi)=H_{\tilde{\nu}_{ \pm}}\left(\frac{ \pm 1-i}{\sqrt{2}} \sqrt{\frac{\mathrm{e} W_{0}}{v_{\mathrm{F}} \hbar}}\left(\xi+\frac{\varepsilon}{\mathrm{e} W_{0}}\right)\right), \quad \tilde{\nu}_{ \pm}=\frac{v_{\mathrm{F}} \hbar}{2 \mathrm{e} W_{0}}\left(-\frac{\mathrm{e} W_{0}}{v_{\mathrm{F}} \hbar} \mp i \frac{\nu^{2}}{R^{2}}\right) .
$$

С учетом соотношений (14), (17) и (19) решение первого из уравнений (16) запишется как

$$
\alpha_{ \pm}(\mathbf{r})=H_{\tilde{\nu}_{ \pm}}\left(\frac{ \pm 1-i}{\sqrt{2}} \sqrt{\frac{\mathrm{e} W_{0}}{v_{\mathrm{F}} \hbar}}\left(\xi+\frac{\varepsilon}{\mathrm{e} W_{0}}\right)\right) \exp \left( \pm i \frac{\left(\varepsilon+\mathrm{e} W_{0} \xi\right)^{2}}{2 \mathrm{e} W_{0} v_{\mathrm{F}} \hbar}+i \mathbf{k}_{\mathrm{F}} \mathbf{r}\right) .
$$

Аналогичным образом записывается решение второго из уравнений (16). Из формулы (20) видно, что вблизи точки Дирака при условии $\left|\mathrm{e} W_{0} \xi\right| \leqslant|\varepsilon|$ набег фазы определяется волновым числом $\mathbf{k}_{\mathrm{F}}$ в точке Ферми.

Решения (20) соответствуют случаю неограниченной УНТ. Данный подход позволяет также получить решение для ГНЛ. В этом случае в (13) условие поперечного квантования $\tilde{\alpha} \sim e^{i \nu \varphi}$ для УНТ нужно заменить на условие квантования ГНЛ $\tilde{\alpha} \sim \sin (2 \pi \nu \eta / H), \nu= \pm 1, \pm 2, \ldots$, где $H$ - ширина ГНЛ, $\eta$ - перпендикулярная оси $O \xi$ координата.

Полученные решения (20) позволяют рассмотреть как частный случай некоторые результаты работы [18], в которой было показано, что для УНТ типа "зигзаг" вблизи точки Дирака уровни энергии квантуются в соответствии с законом

$$
\varepsilon_{s}=\frac{\sqrt{3} \pi \gamma_{0} a}{4 L}(1+2 s)=\frac{v_{\mathrm{F}} \hbar \pi}{2 L}(1+2 s), \quad s=0, \pm 1, \pm 2, \ldots,
$$

a решения $\alpha\left(\mathbf{r}_{p, l}^{A}\right)=\alpha$ и $\beta\left(\mathbf{r}_{p, l}^{A}\right)=\beta$ из (2) имеют вид

$$
\alpha=f \cos \chi+g \sin \chi, \quad \beta=f \sin \chi-g \cos \chi,
$$

где $f, g$ - постоянные величины,

$$
\chi=\frac{\left(\varepsilon+\mathrm{e} W_{0} \xi\right)^{2}}{2 \mathrm{e} W_{0} v_{\mathrm{F}} \hbar} .
$$

В работе [16] $\xi=z,-L / 2 \leqslant z \leqslant L / 2$. Результаты работы [16] можно получить, если учесть, что $E-E_{0} \rightarrow 0$ вблизи поверхности Ферми. В этом случае в (18) $\nu=0$, а второе слагаемое в уравнении (18) по абсолютной величине значительно превосходит первое. Соответственно, получаем, что $Y_{\alpha}(\xi) \sim \xi^{-1 / 2}$, а функции $\alpha_{ \pm}(\mathbf{r})$ пропорциональны $e^{ \pm i \chi}$. Линейная комбинация этих решений приводит к соотношениям (22), где собственные значения энергии определяются равенством (21).

Полученные решения (20) позволяют также рассмотреть условия полной прозрачности потенциальной ямы, в частности парадокс Клейна [10]. Действительно, зависимость фазы (23) от $\xi$ означает, что приращение (набег) фазы на длине УНТ равно $\Delta \chi=\chi(L / 2)-\chi(-L / 2)=\varepsilon L / v_{\mathrm{F}} \hbar$. Если электрон находится в стационарном состоянии, то при таких значениях энергии частицы коэффициент прохождения будет равен нулю, т. е. при $\Delta \chi=\pi(1+2 s) / 2, s= \pm 1, \pm 2, \ldots$, УНТ является непрозрачной системой для электрона. При $\Delta \chi=\pi s, s= \pm 1, \pm 2, \ldots$, УНТ является абсолютно прозрачной системой. Аналогичное условие абсолютной прозрачности 
$\Delta \chi=s_{\mathrm{c}} L=\pi s, s= \pm 1, \pm 2, \ldots$, справедливо для потенциальной ямы прямоугольной формы: коэффициент прохождения записывается как

$$
\tau=\left|\frac{c_{k}}{a_{k}}\right|^{2}=\left(1+\left(\frac{k_{\mathrm{c}}^{2}-k^{2}}{2 k_{\mathrm{c}} s}\right)^{2} \sin ^{2}\left(k_{\mathrm{c}} L\right)\right)^{-1},
$$

где в соответствии с $(20) k_{\mathrm{c}}=\varepsilon / v_{\mathrm{F}} \hbar$.

Условие абсолютной прозрачности $\Delta \chi=\pi s$ имеет место также в случае высокого потенциального барьера (парадокс Клейна [12]), поскольку в случае наличия потенциального барьера вклад в ток проводимости дают дырки (потенциальный барьер для электронов является потенциальной ямой для дырок). Сам парадокс Клейна в ультрарелятивистском случае состоит в том, что безмассовые фермионы Дирака прекрасно передаются через потенциальный барьер независимо от его высоты и ширины [12], [13]. Упрощенный вариант парадокса Клейна был продемонстрирован в однослойной и двуслойной АВ-упаковках биграфена для низкоэнергетических электронов, которые описываются уравнением Дирака для релятивистски безмассовых и массивных фермионов [32]-[34].

Собственные значения энергии и собственные функции состояний УНТ конечной длины могут представлять интерес при создании многокаскадных лазеров [15]-[17], [35]-[40], если в качестве активной области использовать массив параллельно ориентированных наночастиц одинаковой длины порядка 50 нм. Предложения по созданию внутризонных лазеров на квантовых ямах за счет туннельной инжекции туннелирования электронов через потенциальный барьер между квантовыми ямами - появились в 70-х гг. [36] (в работе [37] было показано, что в случае одной квантовой ямы на периоде нельзя создать условия на рабочем переходе, т. е. инверсия обнаружена не была). Идея, предложенная в работе [36], в модифицированном виде используется в квантовых каскадных лазерах. Каскадный лазер был создан, когда стало понятно, что для возникновения инверсии необходимо использовать сверхрешетки с периодом, состоящим из нескольких ям. Среди направлений по созданию ИК-лазеров следует выделить два: создание квантового каскадного лазера на межподзонных переходах [38], [39] и ИК-лазеров на межзонных переходах на основе полупроводниковых гетероструктур с квантовыми ямами и сверхрешетками типа II [40].

\section{4. КОЭФФИЦИЕНТ ПРОХОЖДЕНИЯ ЭЛЕКТРОНОВ ЧЕРЕЗ МАССИВ НЕВЗАИМОДЕЙСТВУЮЩИХ УНТ}

Рассмотрим задачу определения коэффициента прохождения электронов через УНТ или ГНЛ вблизи точки Дирака (именно эти электроны вносят основной вклад в проводимость). Вектор $\mathbf{k}_{\mathrm{F}}$ определяет волновое число и быстроосциллирующую часть полной волновой функции, а решение (13) задает амплитуду волны, слабо зависящей от продольной координаты. Эти величины позволяют однозначно определить коэффициент прохождения [14]. При этом следует подчеркнуть, что в области $|\xi|>L / 2$ поперечные компоненты волнового вектора падающей и прошедшей волн должны совпадать с поперечной компонентой вектора $\mathbf{k}_{\mathrm{F}}$ в области наночастиц. Это означает, что угол хиральности будет определять величину угла падения электронов на торец УНТ. Аналогичная ситуация будет в случае ГНЛ. Исключение составляют УНТ типа "кресло" или ГНЛ типа "зигзаг”, для которых поперечная компонента 
вектора $\mathbf{k}_{\mathrm{F}}$ равна нулю, а волновая функция электрона представляет собой плоскую волну, распространяющуюся вдоль оси УНТ или вдоль ГНЛ.

Пусть волна распространяется слева направо через массив параллельных друг другу невзаимодействующих наночастиц длины $L,-L / 2 \leqslant \xi \leqslant L / 2$. Такую волну представим в виде

$$
\alpha(\xi)=\left\{\begin{array}{lc}
a_{k} e^{i k \xi}+b_{k} e^{-i k \xi}, & \xi<-L / 2, \\
f \alpha_{+}(\xi)+g \alpha_{-}(\xi), & -L / 2 \leqslant \xi \leqslant L / 2, \\
c_{k} e^{i K \xi}, & \xi>L / 2
\end{array}\right.
$$

где $a_{k}, b_{k}$ и $c_{k}$ - амплитуды падающей, отраженной и прошедшей волн $f, g$ - постоянные величины, $\alpha_{ \pm}(\xi)=\alpha_{ \pm}(\mathbf{r}) e^{-i \mathbf{k}_{\mathrm{F}} \mathbf{r}}$, функции $\alpha_{ \pm}(\mathbf{r})$ заданы в $(20)$. При этом потенциальная энергия имеет вид

$$
\widehat{V}=\left\{\begin{array}{lc}
V_{\mathrm{L}}, & \xi<-L / 2, \\
-\mathrm{e} W_{0} \xi, & -L / 2 \leqslant \xi \leqslant L / 2, \\
V_{\mathrm{R}}, & \xi>L / 2
\end{array}\right.
$$

Из условия неразрывности волновой функции $\alpha(\xi)$ и ее производной на границах интервала $-L / 2 \leqslant \xi \leqslant L / 2$ следует система линейных уравнений для коэффициентов. Из этой системы с помощью формулы Крамера нетрудно получить выражение, связывающее амплитуду прошедшей и падающей волны:

$$
\frac{c_{k}}{a_{k}}=\frac{\operatorname{det} \mathcal{C}_{k}}{\operatorname{det} \mathcal{A}_{k}}
$$

где матрицы $\mathcal{C}_{k}$ и $\mathcal{A}_{k}$ имеют вид

$$
\begin{aligned}
& \mathcal{C}_{k}=\left(\begin{array}{cccc}
e^{i k L / 2} & -\alpha_{+}(-L / 2) & -\alpha_{-}(-L / 2) & -e^{-i k L / 2} \\
i k e^{i k L / 2} & \alpha_{+}^{\prime}(-L / 2) & \alpha_{-}^{\prime}(-L / 2) & i k e^{-i k L / 2} \\
0 & \alpha_{+}(L / 2) & \alpha_{-}(L / 2) & 0 \\
0 & \alpha_{+}^{\prime}(L / 2) & \alpha_{-}^{\prime}(L / 2) & 0
\end{array}\right), \\
& \mathcal{A}_{k}=\left(\begin{array}{cccc}
e^{i k L / 2} & -\alpha_{+}(-L / 2) & -\alpha_{-}(-L / 2) & 0 \\
i k e^{i k L / 2} & \alpha_{+}^{\prime}(-L / 2) & \alpha_{-}^{\prime}(-L / 2) & 0 \\
0 & \alpha_{+}(L / 2) & \alpha_{-}(L / 2) & -e^{i K L / 2} \\
0 & \alpha_{+}^{\prime}(L / 2) & \alpha_{-}^{\prime}(L / 2) & -i K e^{i K L / 2}
\end{array}\right) ;
\end{aligned}
$$

здесь штрих означает производную по $\xi$. Из этих формул следует выражение для коэффициента прохождения через УНТ или ГНЛ:

$$
\tau=\frac{K}{k}\left|\frac{c_{k}}{a_{k}}\right|^{2}, \quad \frac{c_{k}}{a_{k}}=\frac{-2 i k\left(\alpha_{+}(L / 2) \alpha_{-}^{\prime}(L / 2)-\alpha_{-}(L / 2) \alpha_{+}^{\prime}(L / 2)\right)}{\Pi e^{i K L / 2+i k L / 2}},
$$

где

$$
\begin{aligned}
\Pi= & \left(\alpha_{+}^{\prime}(-L / 2)+i k \alpha_{+}(-L / 2)\right)\left(\alpha_{-}^{\prime}(L / 2)-i K \alpha_{-}(L / 2)\right)- \\
& -\left(\alpha_{-}^{\prime}(-L / 2)+i k \alpha_{-}(-L / 2)\right)\left(\alpha_{+}^{\prime}(L / 2)-i K \alpha_{+}(L / 2)\right),
\end{aligned}
$$


а $\alpha_{ \pm}$определены в (20). Из формул (25) и (26) следует в частном случае соотношение (24) для коэффициента прохождения волны через потенциальную яму прямоугольной формы [41], для которой

$$
K=k, \quad \alpha_{ \pm}=e^{ \pm i k_{c} \xi}
$$

\section{5. ЗАКЛЮЧЕНИЕ}

На основе модели двухточечной элементарной фазовой ячейки гамильтониан для носителей тока в удлиненных киральных УНТ в окрестности точек Дирака записан в виде двухкомпонентной волновой функции Дирака. Показано, что имеет место эквидистантный спектр собственных значений энергии стационарных состояний, а собственные функции выражаются через полиномы Эрмита. В случае неограниченной УНТ волновое число в основном определяется импульсом в рассматриваемой точке Дирака, а решение выражается через функции Эрмита. Результаты работы применены к задаче определения коэффициента прохождения электронов через массив невзаимодействующих друг с другом УНТ. Полученные результаты можно также применить к массиву нанолент, фуллереновых цепочек и других наночастиц, в частности к карбиновым цепочкам [14].

Если использовать чередующиеся слои из массива УНТ (активная область) и рассматривать слои как потенциальные барьеры, то возникает задача, аналогичная задаче из теории каскадных лазеров [18]. По аналогии с работой [18] из результатов настоящей статьи следует, что массив хиральных нанотрубок можно использовать для решения задачи генерации квазимонохроматического терагерцового излучения (для УНТ длины $L=10^{-7}$ м происходит вынужденный переход с уровня $|k\rangle$ на уровень $|k-1\rangle$, где энергия перехода для УНТ составляет $\Delta \varepsilon=\sqrt{3} \pi \gamma_{0} a \nu / 2 L \approx$ $1.8 \cdot 10^{-2}$ эВ, длина волны перехода $\lambda=2 \pi c / \omega \approx 0.065 \mathrm{Mм}=65$ мкм).

\section{ПРИЛОЖЕНИЕ}

Используя равенство

$$
\mathbf{k r}_{\tilde{p}, \tilde{l}}-\mathbf{k}_{\mathrm{F}} \mathbf{r}_{p, l}=\left(\mathbf{k}-\mathbf{k}_{\mathrm{F}}\right) \mathbf{r}_{\tilde{p}, \tilde{l}}+\mathbf{k}_{\mathrm{F}}\left(\mathbf{r}_{\tilde{p}, \tilde{l}}-\mathbf{r}_{p, l}\right),
$$

преобразуем формулу (10) с учетом (8) к виду

$$
3 \alpha_{p, l}+\sum_{\tilde{p}, \tilde{l}} \alpha_{\tilde{p}, \tilde{l}}=\left\{3 \tilde{\alpha}\left(\mathbf{r}_{p, l}\right)+\sum_{\tilde{p}, \tilde{l}} \tilde{\alpha}\left(\mathbf{r}_{\tilde{p}, \tilde{l}}\right) e^{i \mathbf{k}_{\mathrm{F}}\left(\mathbf{r}_{\tilde{p}, \tilde{l}}-\mathbf{r}_{p, l}\right)}\right\} e^{i \mathbf{k}_{\mathrm{F}} \mathbf{r}_{p, l}} .
$$

Представим выражение в фигурных скобках как

$$
\tilde{\alpha}\left(\mathbf{r}_{p, l}\right)\left\{3+\sum_{\tilde{p}, \tilde{l}} e^{i \mathbf{k}_{\mathrm{F}}\left(\mathbf{r}_{\tilde{p}, \tilde{l}}-\mathbf{r}_{p, l}\right)}\right\}+\sum_{\tilde{p}, \tilde{l}}\left(\tilde{\alpha}\left(\mathbf{r}_{\tilde{p}, \tilde{l}}\right)-\tilde{\alpha}\left(\mathbf{r}_{p, l}\right)\right) e^{i \mathbf{k}_{\mathrm{F}}\left(\mathbf{r}_{\tilde{p}, \tilde{l}}-\mathbf{r}_{p, l}\right)} .
$$

Слагаемое в фигурных скобках в (П.2) в соответствии с равенствами (6) и (9) равно нулю. Разложим в ряд Тейлора выражение $\tilde{\alpha}\left(\mathbf{r}_{\tilde{p}, \tilde{l}}\right)-\tilde{\alpha}\left(\mathbf{r}_{p, l}\right)$ в (П.2). В результате 
с учетом соотношений (П.1) и (П.2) получаем

$$
\begin{aligned}
3 \alpha_{p, l} & +\sum_{\tilde{p}, \tilde{l}} \alpha_{\tilde{p}, \tilde{l}}=\left.\frac{\partial \tilde{\alpha}(\mathbf{r})}{\partial \mathbf{r}}\right|_{\mathbf{r}=\mathbf{r}_{p, l}} e^{i \mathbf{k}_{\mathrm{F}} \mathbf{r}_{p, l}} \sum_{\tilde{p} \tilde{l}}\left(\mathbf{r}_{\tilde{p}, \tilde{l}}-\mathbf{r}_{p, l}\right) e^{i \mathbf{k}_{\mathrm{F}}\left(\mathbf{r}_{\tilde{p}, \tilde{l}}-\mathbf{r}_{p, l}\right)}+ \\
& +\frac{1}{2} \sum_{\tilde{p}, \tilde{l}}\left\{\left.\left(\left(\mathbf{r}_{\tilde{p}, \tilde{l}}-\mathbf{r}_{p, l}\right) \frac{\partial}{\partial \mathbf{r}}\right)^{2} \tilde{\alpha}(\mathbf{r})\right|_{\mathbf{r}=\mathbf{r}_{p, l}} e^{i \mathbf{k}_{\mathrm{F}}\left(\mathbf{r}_{\tilde{p}, \tilde{l}}-\mathbf{r}_{p, l}\right)}\right\} e^{i \mathbf{k}_{\mathrm{F}} \mathbf{r}_{p, l}}
\end{aligned}
$$

В правой части этого равенства первая сумма в соответствии с (6) и (9) равна нулю:

$$
\begin{aligned}
& \sum_{\tilde{p}, \tilde{l}}\left(\mathbf{r}_{\tilde{p}, \tilde{l}}-\mathbf{r}_{p, l}\right) e^{i \mathbf{k}_{\mathrm{F}}\left(\mathbf{r}_{\tilde{p}, \tilde{l}}-\mathbf{r}_{p, l}\right)}= \\
& \quad=-\left.i \frac{\partial}{\partial \mathbf{k}}\left(3+\sum_{\tilde{p}, \tilde{l}} e^{i \mathbf{k}\left(\mathbf{r}_{\tilde{p}, \tilde{l}}-\mathbf{r}_{p, l}\right)}\right)\right|_{\mathbf{k}=\mathbf{k}_{\mathrm{F}}}=-\left.i \frac{\hbar v_{\mathrm{F}}^{2}}{\gamma_{0}^{2}} \frac{\partial\left|\mathbf{p}-\mathbf{p}_{\mathrm{F}}\right|^{2}}{\partial \mathbf{p}}\right|_{\mathbf{p}=\mathbf{p}_{\mathrm{F}}}=0 .
\end{aligned}
$$

Вторую сумму в правой части (П.3), используя соотношения (7) и (9), преобразуем к виду

$$
-\frac{1}{2}\left\{\left.\left(\frac{\partial}{\partial \mathbf{p}} \frac{\partial}{\partial \mathbf{k}}\right)^{2} \tilde{\alpha}_{p, l}(\mathbf{r})\right|_{\mathbf{r}=\mathbf{r}_{p, l}} \sum_{\tilde{p}, \tilde{l}} e^{i \mathbf{k}_{\mathrm{F}}\left(\mathbf{r}_{\tilde{p}, \tilde{l}}-\mathbf{r}_{p, l}\right)}\right\} e^{i \mathbf{k}_{\mathrm{F}} \mathbf{r}_{p, l}}=-\left.\frac{\hbar^{2} v_{\mathrm{F}}^{2}}{\gamma_{0}^{2}} \Delta_{\perp} \tilde{\alpha}_{p, l}(\mathbf{r})\right|_{\mathbf{r}=\mathbf{r}_{p, l}} e^{i \mathbf{k}_{\mathrm{F}} \mathbf{r}_{p, l}} .
$$

Применяя это выражение и равенство (П.3), окончательно получаем

$$
3 \alpha_{p, l}+\sum_{\tilde{p}, \tilde{l}} \alpha_{\tilde{p}, \tilde{l}}=-\left.\frac{\hbar^{2} v_{\mathrm{F}}^{2}}{\gamma_{0}^{2}} \Delta_{\perp} \tilde{\alpha}(\mathbf{r})\right|_{\mathbf{r}=\mathbf{r}_{p, l}} e^{i \mathbf{k}_{\mathrm{F}} \mathbf{r}_{p, l}}
$$

Благодарности. Автор благодарит рецензента за предоставленный список научных работ и уточнение обзора литературы.

\section{Список литературы}

[1] P. R. Wallace, Phys. Rev., 71:9 (1947), 622-634.

[2] K. S. Novoselov, A. K. Geim, S. V. Morozov, D. Jiang, M. I. Katsnelson, I. V. Grigorieva, S. V. Dubonos, A. A. Firsov, Nature, 438 (2005), 197-200.

[3] J. W. McClure, Phys. Rev., 104:3 (1956), 666-671.

[4] A. H. Castro Neto, F. Guinea, N. M. R. Peres, K. S. Novoselov, A. K. Geim, Rev. Modern Phys., 81:1 (2009), 109-162.

[5] J. R. Williams, L. DiCarlo, C. M. Marcus, Science, 317:5838 (2007), 638-641.

[6] V. V. Cheianov, V. Fal'ko, B. L. Altshuler, Science, 315:5816 (2007), 1252-1255.

[7] Т. Я. Тудоровский, А. В. Чаплик, Писъма в ЖЖЭТФ, 84:11 (2006), 735-739.

[8] R. R. Hartmann, N. J. Robinson, M. E. Portnoi, Phys. Rev. B, 81 (2010), 245431, 5 pp.

[9] C. A. Downing, D. A. Stone, M. E. Portnoi, Phys. Rev. B, 84:15 (2011), 155437, 6 pp.

[10] C. J. Tabert, E. J. Nicol, Phys. Rev. B, 86:7 (2012), 075439, 12 pp.

[11] A. L. Rakhmanov, A. V. Rozhkov, A. O. Sboychakov, F. Nori, Phys. Rev. Lett., 109 (2012), 206801, 5 pp.

[12] O. Klein, Z. Phys., 53:3-4 (1929), 157-165.

[13] M. Sanderson, Y.S. Ang, C. Zhang, Phys. Rev. B, 88:24 (2013), 245404, 9 pp. 
[14] P. N. D'yachkov, V. A. Zaluev, E. Yu. Kocherga, N. R. Sadykov, J. Phys. Chem. C, 117:32 (2013), 16306-16315.

[15] A. K. Wojcik, P. Malara, R. Blanchard, T. S. Mansuripur, F. Capasso, A. Belyanin, Appl. Phys. Lett., 103:23 (2013), 231102, 4 pp.

[16] E. Christopher, I. Enobio, K. Ohtani, Y. Ohno, H. Ohno, Appl. Phys. Lett., 103:23 (2013), 231106, 4 pp.

[17] K. Bertling, Y.L. Lim, T. Taimre, D. Indjin, P. Dean, R Weih, S. Höfling, M. Kamp, M. von Edlinger, J. Koeth, A. D. Rakić, Appl. Phys. Lett., 103 (2013), 231107, 3 pp.

[18] Н. Р. Садыков, Н. А. Скоркин, ЖТФ, 83:5 (2013), 1-5.

[19] R. Saito, G. Dresselhaus, M. S. Dresselhaus, Physical Properties of Carbon Nanotube, Imperial College Press, London, 1998.

[20] K. J. A. Reijnders, T. Tudorovskiy, M. I. Katsnelson, Ann. Phys., 333 (2013), 155-197.

[21] O. V. Kibis, D. G. W. Parfitt, M. E. Portnoi, Phys. Rev. B, 71:3 (2005), 035411, 5 pp.

[22] O. V. Kibis, S. V. Malevannyy, L. Hugget, D. G. W. Parfitt, M. E. Portnoi, Electromagnetics, 25:5 (2005), 425-435.

[23] П. Б. Сорокин, Л. А. Чернозатонский, УФН, 183:2 (2013), 113-132.

[24] А.А. Самарский, Теория разностных схем, Наука, М., 1989.

[25] Н. Р. Садыков, Н. А. Скоркин, Оптика атмосферы и океана, $26: 2$ (2013), 160-165.

[26] Н. Р. Садыков, М. О. Садыкова, И. Э. Паршуков, А. Н. Афанасьев, Оптика атмосферы и океана, 19:2-3 (2006), 248-253.

[27] Н. Р. Садыков, Оптика и спектроскопия, 101:6 (2006), 1018-1022.

[28] Н. Р. Садыков, М. О. Садыкова, Оптика атмосферы и океана, 11:2-3 (1998), 223-227.

[29] Н. Р. Садыков, Оптика и спектроскопия, 86:2 (1999), 307-310.

[30] Н. Р. Садыков, Оптика и спектроскопия, 90:5 (2001), 812-816.

[31] А. Ф. Никифоров, В. Б. Уваров, Специальные функиии математической физики, Физматлит, М., 1978.

[32] P. E. Allain, J. N. Fuchs, Eur. Phys. J. B, 83:3 (2011), 301-317, arXiv: 1104.5632.

[33] M. I. Katsnelson, K. S. Novoselov, A. K. Geim, Nature Phys., 2 (2006), 620-625.

[34] J. M. Pereira Jr., F. M. Peeters, A. Chaves, G. A. Farias, Semicond. Sci. Tech, 25:3 (2010), 033002, 9 pp.

[35] L. C. West, S. J. Eglash, Appl. Phys. Lett., 46:12 (1985), 1156-1158.

[36] Р. Ф. Казаринов, Р. А. Сурис, ФТП, 5:4 (1971), 797-800.

[37] M. Helm, P. England, E. Colas, F. DeRosa, S. J. Allen Jr., Phys. Rev. Lett., 63:1 (1989), $74-77$.

[38] Р. Ф. Казаринов, Р. А. Сурис, ФТП, 6:1 (1972), 148-162.

[39] J. Faist, E. Capasso, D. Sivico, C. Sirtori, A. L. Hutchinson, A. Y. Cho, Science, 264:5158 (1994), 553-556.

[40] B. H. Yang, D. Zhang, R. Q. Yang, C.-H. Lin, S. J. Murry, S. S. Pei, Appl. Phys. Lett., 72:18 (1998), 2220-2222.

[41] Л. Д. Ландау, Е. М. Лифшиц, Теоретическая физика, т. 3: Квантовая механика. Нерелятивистская теория, Наука, М., 2002.

Поступила в редакцию 18.01.2014, после доработки 17.03.2014 